\title{
Ultrafast spin-transfer switching in spin valve nanopillars with perpendicular anisotropy
}

Cite as: Appl. Phys. Lett. 96, 022514 (2010); https://doi.org/10.1063/1.3284515

Submitted: 30 October 2009 . Accepted: 10 December 2009. Published Online: 14 January 2010

D. Bedau, H. Liu, J.-J. Bouzaglou, A. D. Kent, J. Z. Sun, J. A. Katine, E. E. Fullerton, and S. Mangin

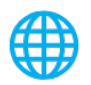

\section{ARTICLES YOU MAY BE INTERESTED IN}

Spin-transfer pulse switching: From the dynamic to the thermally activated regime Applied Physics Letters 97, 262502 (2010); https://doi.org/10.1063/1.3532960

Ultrafast switching in magnetic tunnel junction based orthogonal spin transfer devices Applied Physics Letters 97, 242510 (2010); https://doi.org/10.1063/1.3527962

Spin torque switching of perpendicular $\mathrm{Ta}|\mathrm{CoFeB}| \mathrm{MgO}$-based magnetic tunnel junctions Applied Physics Letters 98, 022501 (2011); https://doi.org/10.1063/1.3536482

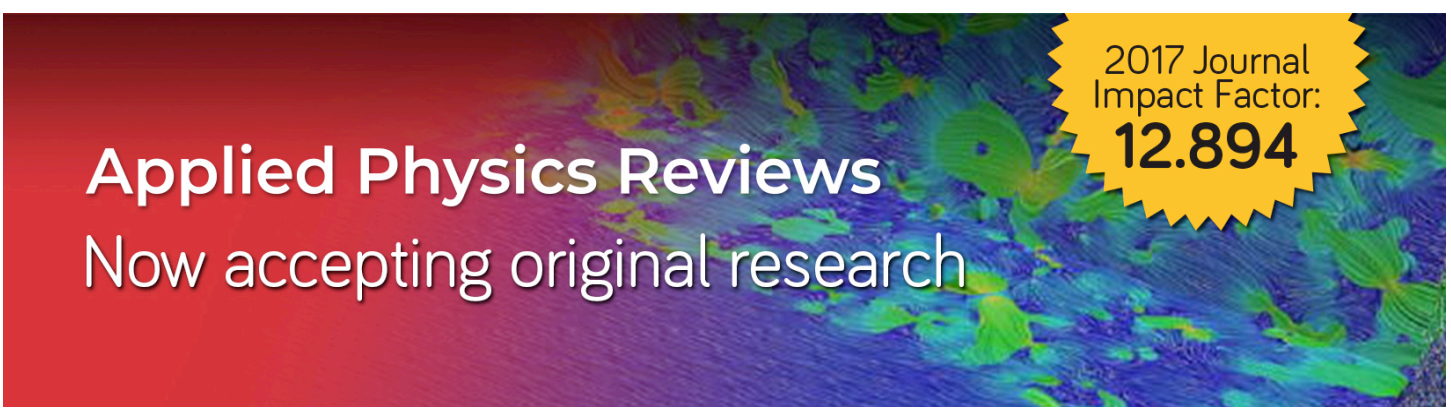




\title{
Ultrafast spin-transfer switching in spin valve nanopillars with perpendicular anisotropy
}

\author{
D. Bedau, ${ }^{1, a)}$ H. Liu, ${ }^{1}$ J.-J. Bouzaglou, ${ }^{1,5}$ A. D. Kent, ${ }^{1}$ J. Z. Sun, ${ }^{2}$ J. A. Katine, ${ }^{3}$ \\ E. E. Fullerton, ${ }^{4}$ and S. Mangin ${ }^{5}$ \\ ${ }_{1}^{1}$ Department of Physics, New York University, 4 Washington Place, New York, New York 10003, USA \\ ${ }^{2}$ IBM T. J. Watson Research Center, P.O. Box 218, Yorktown Heights, New York 10598, USA \\ ${ }^{3}$ San Jose Research Center, Hitachi-GST, San Jose, California 95135, USA \\ ${ }^{4}$ CMRR, University of California, San Diego, La Jolla, California 92093-0401, USA \\ ${ }^{5}$ IJL, Nancy-Université, UMR CNRS 7198, F-54042 Vandoeuvre Cedex, France
}

(Received 30 October 2009; accepted 10 December 2009; published online 14 January 2010)

Spin-transfer switching with short current pulses has been studied in spin-valve nanopillars with perpendicularly magnetized free and reference layers. Magnetization switching with current pulses as short as $300 \mathrm{ps}$ is demonstrated. The pulse amplitude needed to reverse the magnetization is shown to be inversely proportional to the pulse duration, consistent with a macrospin spin-transfer model. However, the pulse amplitude duration switching boundary depends on the applied field much more strongly than predicted by the zero temperature macrospin model. The results also demonstrate that there is an optimal pulse length that minimizes the energy required to reverse the magnetization. (C) 2010 American Institute of Physics. [doi:10.1063/1.3284515]

Spin-transfer devices hold great promise for achieving fast and high density nonvolatile magnetic random-access memory (ST-MRAM). ${ }^{1}$ There has been an intense effort world-wide to reduce the switching current and the switching time, while maintaining thermally stable nanometer scale magnetic elements. ${ }^{2-6}$ Devices with perpendicular anisotropy show great promise in this respect, since the high anisotropy may lead to stable elements at room temperature, even at sub $10 \mathrm{~nm}$ lateral sizes. In addition, the spin-transfer figure of merit, the ratio of the threshold current for switching to the energy barrier to thermal reversal, has been shown to be small in all-perpendicularly magnetized spin-valve nanopillars, ${ }^{7}$ as expected based on theoretical models. ${ }^{8}$ Recently switching currents on the order of $100 \mu \mathrm{A}$ have been observed in quasistatic studies of $50 \mathrm{~nm}$ diameter nanopillars at room temperature. ${ }^{7}$

A key issue for memory applications is the possibility of fast, energy-efficient switching under short current pulses. Several groups have reported magnetization dynamics on short time scales in nanopillars with in-plane magnetized elements. ${ }^{9-13}$ Short-time switching has not been studied thus far in perpendicularly magnetized layers. However, results have been reported for devices with an in-plane free layer and a perpendicular polarizer. ${ }^{14-16}$

In this letter, we present studies of ultrafast spin-transfer switching in all-perpendicularly magnetized spin-valve nanopillars. We measure the probability of switching for short (100 ps-10 ns) current pulses as a function of pulse amplitude and duration. We also examine how the amplitudeduration boundaries depend on the applied perpendicular magnetic field and find a simple relation between the pulse amplitude and duration.

Our experiments were conducted on multilayer spinvalves fashioned into nanopillars that contain two magnetic layers, a reference and a free layer, both with perpendicular magnetic anisotropy, separated by a thin $\mathrm{Cu}$ spacer layer. The

${ }^{a)}$ Electronic mail: db137@nyu.edu. free layer is a $\mathrm{Co} / \mathrm{Ni}$ multilayer while the reference layer is designed to have higher magnetic anisotropy and coercivity than the free layer. It consists of a composite of $\mathrm{Co} / \mathrm{Pt}$ and $\mathrm{Co} / \mathrm{Ni}$ multilayers. The layer stack is $\mathrm{Ta}(5) / \mathrm{Cu}(30) / \mathrm{Pt}(3)$ $\times[\mathrm{Co}(0.25) / \mathrm{Pt}(0.52)] \times 4 / \mathrm{Co}(0.25) /[\mathrm{Ni}(0.6) / \mathrm{Co}(0.1)] \times 2 /$ $\mathrm{Cu}(4) /[\mathrm{Co}(0.1) / \mathrm{Ni}(0.6)] \times 2 / \mathrm{Co}(0.2) / \mathrm{Pt}(3) / \mathrm{Cu}(20) / \mathrm{Ta}(5)$ (layer thickness in nanometer), similar to samples used in Ref. 7. The samples were patterned combining e-beam and optical lithography. The sample sizes studied were $50 \times 50$, $100 \times 100$, and $100 \times 150 \mathrm{~nm}^{2}$. In total 19 samples were studied. The field was always applied perpendicular to the film plane, i.e., along the easy anisotropy axis. Before starting an experiment the reference layer was saturated with a perpendicular field of about $0.5 \mathrm{~T}$. During pulse injection the applied fields do not exceed $200 \mathrm{mT}$, and do not affect the magnetic state of the reference layer. All measurements were performed at room temperature.

The samples have two pairs of contacts to the top and bottom electrode. On both contact pairs bias tees were used to split the high and low frequency circuits. The first contact pair was connected to a pulse generator and the second pair to an oscilloscope to record the transmitted waveform. Through the dc port of the bias tee, a low frequency current of $300 \mu \mathrm{A}_{\mathrm{rms}}$ was injected into the sample to measure the ac resistance using a standard lock-in technique. While measuring the ac resistance the sample could be subjected both to short time pulses as well as to dc currents. A conventional current flowing from the bottom to the top layer is defined as positive.

By measuring the resistance for all possible contact configurations, an equivalent circuit model was generated that allows relating the pulse amplitude to the current through the spin valve. Replacing the pulse generator with a microwave source and measuring the amplitude of the transmitted signal with the oscilloscope, we find that the measured transmission parameters agree with the circuit model with a few percent accuracy. 

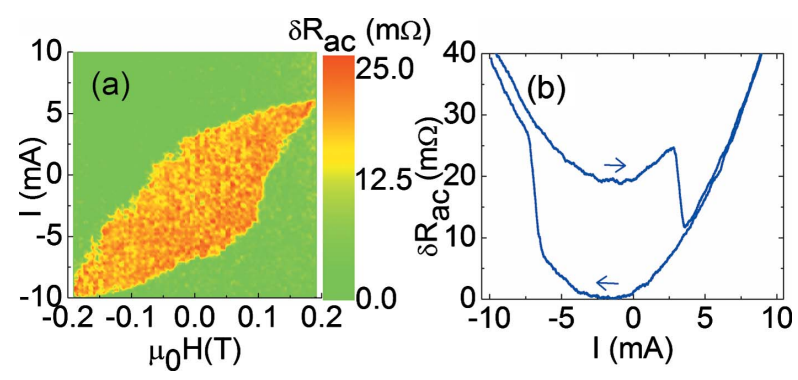

FIG. 1. (Color online) (a) Phase Diagram of a $100 \times 100 \mathrm{~nm}^{2}$ nanopillar spin valve. The resistance obtained for decreasing currents was subtracted from the resistance obtained for increasing currents to make the metastable region visible. (b) Zero field current sweep. For both measurements the field was fixed while the current was swept at a rate of $200 \mu \mathrm{A} / \mathrm{s}$. The junction resistance is $6.6 \Omega$.

To determine the behavior of the sample in the quasistatic case, the dc current was swept for a series of fixed fields. To determine the dynamic behavior of the samples, pulses between $100 \mathrm{ps}$ and $10 \mathrm{~ns}$ duration and amplitudes of up to $2 \mathrm{~V}$ were injected while maintaining a constant external magnetic field. After each pulse injection the ac resistance was measured to determine whether a switching event had occurred. Each pulse injection was repeated 100 to 10000 times to obtain the switching probability.

Figure 1(a) shows the switching phase diagram of a typical $100 \times 100 \mathrm{~nm}^{2}$ nanopillar. The color in this diagram is the resistance obtained during the down sweep subtracted from the one from the up sweep. This type of phase diagram illustrates the bistable region for which both parallel $(\mathrm{P})$ and antiparallel (AP) magnetization configurations coexist. Figure 1(b) shows a zero field current sweep across the hysteretic region. The critical current to switch from $\mathrm{P}$ to AP is 6.8 $\mathrm{mA}$ and from AP to $\mathrm{P}$ is $3.2 \mathrm{~mA}$, and the magnetoresistance is $0.3 \%$. We note that for these quasistatic measurements we expect lower switching fields and switching currents than for short pulses due to the longer time available for thermally assisted reversal.

From the width of the AP region obtained for a field sweep at zero current we can determine the switching field to be $95 \mathrm{mT}$. We note that the field-swept hysteresis loop is not centered at zero field. This is associated with dipolar interactions between the reference and free layers. The loop shift is $16 \mathrm{mT}$.

For the following analysis we will focus on the AP to $\mathrm{P}$ transition. The $\mathrm{P}$ to AP transition has been shown to give similar results. The switching probability as a function of pulse duration and amplitude is shown in Fig. 2(a). For low current durations/amplitudes the switching probability is zero (blue), whereas for longer durations or amplitudes the switching probability reaches one (red). The black curve depicts a switching probability of 50\% and defines the boundary between the P and AP states. Magnetization switching for pulse durations down to $300 \mathrm{ps}$ is observed.

We now describe the behavior in the framework of a zero temperature macrospin model. Starting from the Landau-Lifshitz-Gilbert (LLG) equation with spin torque, $\mathrm{Sun}^{8}$ linearized the equations around the initial angle to determine the limits of the stability in the small cone angle limit. Sun then equated the onset of instability with the occurrence of a switching event, obtaining both the critical current as well as the switching time. The predicted zero tem-
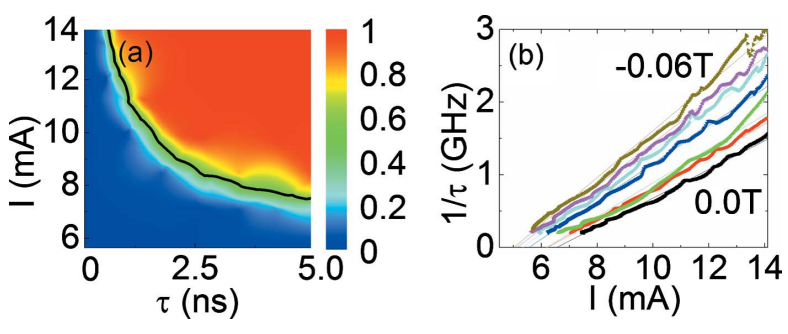

FIG. 2. (Color online) (a) Switching probability as a function of the duration and amplitude of the current pulse for zero applied field. The 50\% switching probability line is shown in black. (b) $50 \%$ switching probability for different applied fields $\mu_{0} H$ from 0 to $-60 \mathrm{mT}$.

perature critical current for AP-P $\left(I_{\mathrm{c} 0+}\right)$ and P-AP switching $\left(I_{\mathrm{c} 0-}\right)$ has the following form:

$$
I_{\mathrm{c} 0 \pm}=\frac{\alpha}{p g_{ \pm}} \frac{2 e}{\hbar} M_{\mathrm{s}} V \mu_{0}\left(H \pm H_{\mathrm{K}}\right)
$$

where $\alpha$ is the damping and $p$ is the spin-polarization of the current. $g(\theta)$ describes the angular variation in the spin torque, for AP to P switching $g_{+}=g(\pi)$ and correspondingly $g_{-}=g(0)$ for P to AP switching.

Alternatively to the linearization, ${ }^{8}$ the full LLG equation can be integrated up to the time when the critical angle is reached, equating this time with the switching time. Both the linearization in the small cone angle limit as well as the full solution of the LLG equation lead to a switching time of the following form:

$$
1 / \tau=A\left(I-I_{\mathrm{c} 0}\right) .
$$

The parameter $A$, which we denote the dynamic parameter, governs the switching rate. Plotting the $50 \%$ switching probability boundary of Fig. 2(a) after a change of coordinates, we see that the boundary is indeed linear, Fig. 2(b).

From the slopes and the intercepts of the $50 \%$ boundaries for different applied magnetic fields the dynamic parameter $\mathrm{A}$, as well as the extrapolated critical currents were obtained, shown in Fig. 3. The critical current varies linearly with the applied external field, as is expected from the macrospin model. The zero temperature/zero field critical current extrapolated from Fig. 2(b) has a value of $6.6 \mathrm{~mA}$. This value is twice as high as the room temperature critical current determined for longer timescales [Fig. 1(b)], $3.2 \mathrm{~mA}$. Indeed, when the pulse duration is short enough, the magnetization dynamics enter a "dynamic regime" for which thermal fluctuations do not perturb the switching trajectory but only set the initial magnetization configuration. ${ }^{17}$

Figure 3 shows the dynamic parameter A and the critical current determined from the curves in Fig. 2(b). Green disks correspond to the measured dynamic parameters, while red triangles correspond to the measured switching currents. The curves are computed from the macrospin model. From the corresponding equations we can extract the coercive field $\mu_{0} H_{\mathrm{K}}=245 \mathrm{mT}$, the damping $\alpha=0.011$, the spin polarization $P=0.015$ and $\theta_{0}=1.36 \mathrm{rad}$, the initial angle of the macrospin with respect to the $z$ axis. The ratio $\alpha / \mathrm{P}=0.77$ and $\mu_{0} H_{\mathrm{K}}$ are close to the values determined from the quasistatic measurements, but the initial angle is unphysically large and the spin polarization is unphysically small. For an initial angle of $\theta_{0}=0.05 \mathrm{rad}$, determined from a Boltzmann distribution, the field dependence of A (dashed line) cannot explain the strong 


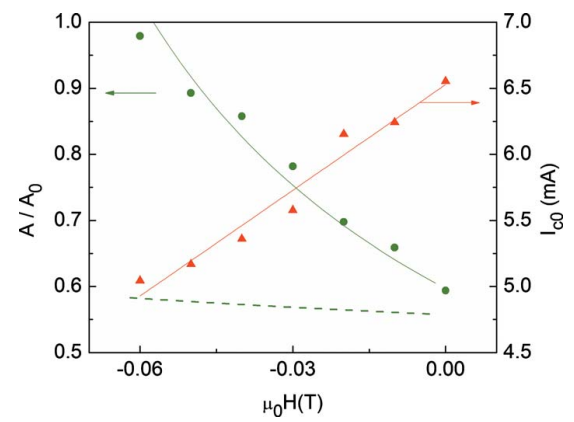

FIG. 3. (Color online) Field dependence of the dynamic parameter A and the critical current on the external field. The dots and triangles are experimental results, while the lines are obtained from the macrospin model for different parameters. The solid lines are a fit corresponding to $\mu_{0} H_{\mathrm{K}}=245 \mathrm{mT}, \alpha$ $=0.011, P=0.015$, and $\theta_{0}=1.36 \mathrm{rad}$. The experimentally found strong dependence of the dynamic parameter on field manifests itself in a large initial angle. Assuming a Boltzmann distribution, we obtain an average initial angle of $0.05 \mathrm{rad}$, leading to the dashed curve. The dynamic parameters for the different curves have been normalized with the following coefficients: $A_{0, \operatorname{Exp}}=3.3 \times 10^{11} \mathrm{~s}^{-1} \mathrm{~A}^{-1}, \quad A_{0, \theta_{0}=1.36}=10^{13} \mathrm{~s}^{-1} \mathrm{~A}^{-1}, \quad$ and $A_{0, \theta_{0}=0.05}=5$ $\times 10^{10} \mathrm{~s}^{-1} \mathrm{~A}^{-1}$.

variation with field of the measured $\mathrm{A}$. The predicted reversal rate A depends strongly on the initial angle. For an assumed initial angle of $\theta_{0}=1.36 \mathrm{rad}$ the macrospin model predicts a rate 30 times larger than the experimentally determined value, for $\theta_{0}=0.05 \mathrm{rad}$ the predicted rate is lower than the experimentally obtained value by a factor of 10 .

The macrospin model predicts a zero temperature energy barrier to reversal proportional to the coercive field

$$
U_{\mathrm{K}}=M_{\mathrm{S}} V \mu_{0} H_{\mathrm{K}} / 2 .
$$

From the coercive field $\mu_{0} H_{\mathrm{K}}=245 \mathrm{mT}$, determined using Eq. (1), we obtain a zero temperature energy barrier of $8.7 \mathrm{eV}$ or $338 \mathrm{kT}$. The spin-transfer figure of merit therefore has a value of $19 \mu \mathrm{A} / \mathrm{kT}$. Using the zero temperature coercive field and the energy barrier, we can estimate the room temperature coercive field assuming the magnetization relaxation rate obeys an Arrhenius law. ${ }^{\top}$ We find a room temperature coercive field of $180 \mathrm{mT}$, about two times larger than the measured value.

From the relationship between pulse duration and current given in Eq. (2) we can determine the energy requirement for switching as a function of the pulse duration, $E$ $\propto I^{2} \tau$. For short times the energy increases with $1 / \tau$, while for long pulse durations the energy is proportional to $\tau$, with an optimum at $1 /\left(A I_{\mathrm{c} 0}\right) \approx 770$ ps for $\mu_{0} H=0$ and $I_{\mathrm{c} 0}$ $=6.6 \mathrm{~mA}$. Experimentally, we find an optimal pulse duration of $660 \mathrm{ps}$, requiring $E=0.9 \mathrm{pJ}$, five times less than for a pulse of $10 \mathrm{~ns}$ duration. Applying higher amplitudes the switching probability at $660 \mathrm{ps}$ is $100 \%$. These results show reliable sub-nsec pulse switching with low energy consumption in all perpendicular devices, making them promising candidates for ST-MRAM.

In conclusion, we have demonstrated ultrafast switching in an all-perpendicular spin valve with high efficiency. We have analyzed the behavior in the framework of the single domain model and find a general agreement. The amplitude needed for switching is proportional to $1 / \tau$, as predicted by the macrospin model. We find a linear dependence of the critical current and dynamic parameter on the applied easyaxis field as expected for the case of a uniaxial anisotropy. Even though the switching process follows the predicted scaling law, the macrospin model does not account for the material parameters. The model cannot explain the strong damping and predicts a very low spin polarization and an initial angle which is far too large. Possible explanations are that the reversal process occurs by domain wall nucleation or propagation and that there is additional dissipation associated with the excitation of spin-waves.

The research at NYU was supported by USARO (Grant No. W911NF0710643).

${ }^{1}$ J. A. Katine and E. E. Fullerton, J. Magn. Magn. Mater. 320, 1217 (2008). ${ }^{2}$ D. Chiba, Y. Sato, T. Kita, F. Matsukura, and H. Ohno, Phys. Rev. Lett. 93, 216602 (2004)

${ }^{3}$ P. M. Braganca, I. N. Krivorotov, O. Ozatay, A. G. F. Garcia, N. C. Emley, J. C. Sankey, D. C. Ralph, and R. A. Buhrman, Appl. Phys. Lett. 87, 112507 (2005)

${ }^{4}$ O. Ozatay, P. G. Gowtham, K. W. Tan, J. C. Read, K. A. Mkhoyan, M. G. Thomas, and G. D. Fuchs, Nature Mater. 7, 567 (2008).

${ }^{5}$ S. Mangin, Y. Henry, D. Ravelosona, J. A. Katine, and E. E. Fullerton, Nature Mater. 5, 210 (2006).

${ }^{6}$ T. Devolder, J. Hayakawa, K. Ito, H. Takahashi, S. Ikeda, P. Crozat, N. Zerounian, J.-V. Kim, C. Chappert, and H. Ohno, Phys. Rev. Lett. 100, 057206 (2008)

S. Mangin, Y. Henry, D. Ravelosona, J. A. Katine, and E. E. Fullerton, Appl. Phys. Lett. 94, 012502 (2009).

${ }^{8}$ J. Z. Sun, Phys. Rev. B 62, 570 (2000).

${ }^{9}$ A. A. Tulapurkar, T. Devolder, K. Yagami, P. Crozat, C. Chappert, A. Fukushima, and Y. Suzuki, Appl. Phys. Lett. 85, 5358 (2004).

${ }^{10}$ S. Kaka, M. R. Pufall, W. H. Rippard, T. J. Silva, S. E. Russek, J. A Katine, and M. Carey, J. Magn. Magn. Mater. 286, 375 (2005).

${ }^{11}$ M. L. Schneider, M. R. Pufall, W. H. Rippard, S. E. Russek, and J. A Katine, Appl. Phys. Lett. 90, 092504 (2007).

${ }^{12}$ T. Devolder, C. Chappert, J. A. Katine, M. J. Carey, and K. Ito, Phys. Rev. B 75, 064402 (2007).

${ }^{13}$ S. Garzon, L. Ye, R. A. Webb, T. M. Crawford, M. Covington, and S. Kaka, Phys. Rev. B 78, 180401 (2008).

${ }^{14}$ O. J. Lee, V. S. Pribiag, P. M. Braganca, P. G. Gowtham, D. C. Ralph, and R. A. Buhrman, Appl. Phys. Lett. 95, 012506 (2009).

${ }^{15}$ C. Papusoi, B. Delaet, B. Rodmacq, D. Houssameddine, J.-P. Michel, U. Ebels, R. C. Sousa, L. Buda-Prejbeanu, and B. Dieny, Appl. Phys. Lett. 95, 072506 (2009)

${ }^{16}$ J.-M. L. Beaujour, D. Bedau, H. Liu, M. R. Rogosky, and A. D. Kent, Proc. SPIE 7398, 73980D (2009).

${ }^{17}$ J. Z. Sun, T. S. Kuan, J. A. Katine, and R. H. Koch, Proc. SPIE 5359, 445 (2004). 